\title{
Blockade of the hedgehog pathway inhibits osteophyte formation in arthritis
}

\author{
Gisela Ruiz-Heiland, ${ }^{1}$ Angelika Horn, ${ }^{1}$ Pawel Zerr, ${ }^{1}$ Willy Hofstetter, ${ }^{2}$ Wolfgang Baum, ${ }^{1}$ \\ Michael Stock, ${ }^{1}$ Jörg H Distler, ${ }^{1}$ Falk Nimmerjahn, ${ }^{3}$ Georg Schett, ${ }^{1}$ Jochen Zwerina ${ }^{1,4}$
}

\begin{abstract}
- Additional data (supplementary figure and table) are published online only. To view these files please visit the journal online (http://ard. bmj.com/content/71/3.toc)

${ }^{1}$ Department of Internal Medicine 3 and Institute for Clinical Immunology, University of Erlangen-Nurnberg, Erlangen, Germany

${ }^{2}$ Group for Bone Biology, Department of Clinical Research, University of Bern, Bern, Switzerland ${ }^{3}$ Department of Genetics, University of Erlangen-Nurnberg, Erlangen, Germany

${ }^{4}$ Ludwig Boltzmann Institute of Osteology, Hanusch Hospital, Vienna, Austria
\end{abstract}

\section{Correspondence to}

Jochen Zwerina, Department of Internal Medicine 3 and Institute for Clinical Immunology, University of ErlangenNuremberg, Krankenhausstrasse 12, D-91054 Erlangen, Germany;

jochen.zwerina@uk-erlangen.de

Received 14 December 2010 Accepted 12 June 2011 Published Online First 10 January 2012

\begin{abstract}
Background Osteophyte formation is a common phenomenon in arthritis. Bone formation by endochondral ossification is considered a key pathophysiological process in the formation of osteophytes.

Objective To examine the hypothesis that inhibition of smoothened (Smo), a key component of the hedgehog pathway inhibits osteophyte formation as the hedgehog pathway mediates endochondral ossification.

Methods Arthritis was induced in 8-week-old C57/BL6 mice by serum transfer (K/BxN model). Mice were then treated by daily administration of either vehicle or LDE223, a specific small molecule inhibitor for Smo, over 2 weeks starting at the onset of disease. Clinical course of arthritis, histological and molecular changes of bone in the affected joints as well as systemic bone changes were assessed.

Results Serum transfer-induced arthritis led to severe osteophyte formation within 2 weeks of onset. Blockade of Smo inhibited hedgehog signalling in vivo and also significantly inhibited osteophyte formation, whereas the clinical and histopathological signs of arthritis were not affected. Also, systemic bone mass did not change. Smo inhibitor particularly blocked the formation of hypertrophic chondrocytes and collagen type $\mathrm{X}$ expression.

Conclusions The data indicate that blockade of hedgehog signalling by targeting Smo specifically inhibits osteophyte formation in arthritis without affecting inflammation and without eliciting bone destruction at the local and systemic level. Blockade of Smo may thus be considered as a strategy to specifically influence the periosteal bone response in arthritis associated with bone apposition.
\end{abstract}

\section{INTRODUCTION}

Inflammatory and degenerative joint diseases frequently elicit proliferative responses of the periarticular periosteal bone, leading to the formation of bony spurs (osteophytes). In inflammatory arthritis, such as psoriatic arthritis and spondyloarthritis, osteophytes or syndesmophytes originate at the bone cartilage border or tendon insertion sites (entheses), respectively, in peripheral joint and the vertebral bodies. Within this process periosteal cells and cells located in the entheses appear to be primarily involved. Areas of fibrocartilage at the insertion sites of the tendons are particularly sensitive to osteophyte formation and mechanical forces have been considered as important triggers for the onset of these lesions. ${ }^{1}$ Osteophytes and syndesmophytes can lead to ankylosis with impairments of motion and joint dysfunction. It is therefore of central importance to understand the molecular mechanisms, which drive bony spur formation to develop strategies to prevent them.

The mechanism of osteophyte formation, however, is incompletely understood. ${ }^{2-4}$ Osteophytes are typically formed by endochondral ossification: mesenchymal cells undergo condensation and start chondrogenic differentiation, going through different stages and thereby progressively expressing collagen type II, later becoming prehypertrophic and hypertrophic cells expressing collagen type X. The latter cells also express matrix metalloproteinase 13 and vascular endothelial growth factor. This results in the formation of vessels and invasion of osteoclasts that break down the calcified matrix and attract osteoblast precursors, leading to bone formation. Within this complex process the replacement of hypertrophic cartilage by bone and also the steering effect of border hypertrophic chondrocytes may play a role. ${ }^{5}$ Bone morphogenic proteins (BMPs) as well as Wnt proteins (Wnts) appear to support osteophyte formation as both pathways are crucially involved in osteoblast differentiation. Their influence on the chondrocyte differentiation is, however, different as BMPs support early differentiation of progenitor cells into chondroblasts, whereas some Wnts may have an inhibitory effect on chondrocyte differentiation. ${ }^{6-8}$ Strategies blocking BMP or Wnt proteins successfully suppress osteophyte formation but may also affect bone formation in general, as osteoblasts physiologically depend on these two pathways. In contrast, hypertrophic chondrocytes are part of endochondral ossification and typically localised in the growth plates but are not involved in physiological turnover of the adult skeleton. Hypertrophic chondrocytes are abundantly and consistently present in osteophytes of mouse models of inflammatory arthritis and human lesions.

$\mathrm{Hh}$ is a protein family consisting of sonic hedgehog (Shh), Indian hedgehog (Ihh) and desert hedgehog (Dhh) which plays a crucial role during embryonic development by governing growth through the development of chondrocytes and promoting endochondral ossification..$^{9-11}$ Ligation of Hh to the Hh receptor patched 1 (Ptch1) leads to its dissociation from its key component smoothened (Smo). Smo a G protein-coupled receptorlike molecule positively regulates $(\mathrm{Hh})$ signal 
transduction by activating several downstream transcription factors termed glioma-associated-oncogen-homologues (Gli1, Gli2, Gli3), which induce the transcription of Hh-dependent genes. ${ }^{12}{ }^{13}$ Activation of Hh signalling is especially important for chondrocyte hypertrophy during development. Also postnatally $\mathrm{Hh}$ appears to drive chondrocyte hypertrophy. Thus, pathological chondrocyte hypertrophy in the osteoarthritic articular cartilage has been linked to Hh signalling. ${ }^{14}$ However, it is not clear whether the same mechanisms apply to osteophyte formation.

Based on its key role in the differentiation of hypertrophic chondrocytes and the abundance of these cells in osteophytes we speculated that the Hh pathway contributed to osteophyte formation of arthritis. In this study, we blocked Hh by targeting its essential component Smo in an experimental mouse model of inflammatory arthritis.

\section{MATERIAL AND METHODS}

Animals and treatment

Eight-week-old male C57/BL6 mice were used for this study. Animals were divided into three groups ( $n=6 /$ group): In groups 1 and 2, arthritis was induced by intraperitoneal injection of $300 \mu \mathrm{l}$ serum from $\mathrm{K} / \mathrm{BxN}$ mice as described previously. ${ }^{15}$ The third group was left untreated and served as negative control group. Three days after arthritis induction, animals were treated according to the following protocol: group 1 received the selective orally bioavailable Smo antagonist LDE223 (40 $\mathrm{mg} / \mathrm{kg} /$ day; obtained from Novartis, Basel, Switzerland ${ }^{16}$; dissolved in 10\% dimethyl sulphoxide (DMSO) in polyethylene glycol-300 (PEG-300) and group 2 received the vehicle $(10 \%$ DMSO in PEG-300) only. LDE223 is a potent selective orthobiphenylcarboxamide structure Smo antagonist with an IC50 of $11 \mathrm{nM}$ (at $1 \mathrm{mM}$ Hh agonist) in a Gli luciferase activity shift assay and with an IC50 of $55 \mathrm{nM}$ in a Smo binding assay using Bodipy cyclopamine competition. Treatments were administered once daily in a total volume of $100 \mu$ by oral gavage. Vehicle (10\% DMSO in PEG-300) treatment itself did not have any effects on clinical and histological signs of arthritis when results were compared with phosphate-buffered saline-treated mice (data not shown). After 2 weeks, animals were killed by cervical dislocation under general anaesthesia. The local ethics committee of the University of Erlangen-Nuremberg approved the animal procedures.

\section{Assessment of arthritis}

Mice were assessed for body weight and the clinical signs of arthritis were semiquantitatively evaluated as described previously: Briefly, joint swelling was examined in all four paws, and a clinical score of $0-3$ was assigned ( $0=$ no swelling, $1=$ mild, $2=$ moderate and $3=$ severe swelling of the toes and ankle). In addition, grip strength was examined in each paw, using a $3 \mathrm{~mm}$ diameter wire, and was scored on a scale of 0 to $-4(0=$ normal grip strength, $-1=$ mildly reduced, $-2=$ moderately reduced, $-3=$ severely reduced and $-4=$ no grip strength). ${ }^{8}$

\section{Histological analysis and bone histomorphometry}

Left and right paws were fixed overnight in $4.0 \%$ formalin and then decalcified with EDTA, embedded in paraffin and stained with haematoxylin and eosin (H\&E), tartrate-resistant acid phosphatase (TRAP) and toluidine blue for histological assessment of articular inflammation, proteoglycan loss, bone erosions and osteophyte formation. Analysis was performed using a microscope (Nikon, Berlin, Germany) equipped with a video camera and digital analysis system (OsteoMeasure; OsteoMetrics, Decatur, GA, USA).

For determination of cartilage proteoglycan loss the areas of total cartilage and non-labelled cartilage were measured in sections stained with toluidine blue, as described previously. ${ }^{17}$ The areas of inflammation and bone erosions were evaluated in H\&E-stained sections as the sum of the areas of inflammation and erosions, respectively, in all digital, carpal and tarsal joints. Osteoclasts were assessed by counting multinucleated TRAP-positive cells in each paw section. ${ }^{18}$ Hypertrophic chondrocytes within osteophytes were identified by their characteristic morphology and were counted on toluidine blue-stained hind paw sections. Quantification was performed by relating the number of hypertrophic chondrocytes to total cell number within osteophytes. Osteophyte-covered bone surface was also determined by histomorphometry by measuring the length of periosteal bone surface covered by osteophytes and relating it to the length of total periosteal bone surface.

Trabecular bone architecture was measured using undecalcified plastic-embedded methylmethacrylate sections of tibial bones (Technovit; Heraus Kulzer, Wehrheim, Germany). Sections (3-4 $\mu \mathrm{m})$ were stained with von Kossa and Goldner for the analysis of structural parameters of the trabecular bone by histomorphometry. Bone volume per total volume, trabecular number and trabecular thickness were measured as recommended by the American Society of Bone and Mineral Research. ${ }^{19}$

\section{Dynamic labelling of bone}

Bone formation was assessed by dynamic histomorphometry. Calcein (30 mg/kg body weight, Sigma-Aldrich, St. Louis, $\mathrm{MO}$, US) was injected subcutaneously 9 and 2 days before mice were sacrificed. The entire bone marrow region of the left tibia (trabecular compartment) and the periosteal bone of the tibia were separately assessed by fluorescence microscopy and mineral apposition rate ( $\mu \mathrm{m} /$ day) was determined by histomorphometry.

\section{Immunohistochemistry}

Tissues were carefully prepared, fixed in paraformaldehyde and embedded in paraffin. Sections $(2-3 \mu \mathrm{m})$ were deparaffinised, hydrated and incubated with hyaluronidase (Roche, Grenzach, Germany) for antigen retrieval. Samples were incubated overnight with a polyclonal rabbit antiperiostin antibody (ab14041, Abcam, Cambridge, MA, USA) at a concentration of $5 \mu \mathrm{g} / \mathrm{ml}$, or a polyclonal rabbit anti-Gli2 antibody (ab7195, Abcam) at a concentration of $10 \mu \mathrm{g} / \mathrm{ml}$ or a monoclonal mouse anticollagen type $\mathrm{X}$ antibody (provided by Klaus von der Mark, Erlangen, Germany) at a concentration of $10 \mu \mathrm{g} / \mathrm{ml}$. As negative control normal rabbit or mouse IgG (Dako, Vienna, Austria) at a concentration of $10 \mu \mathrm{g} / \mathrm{ml}$ was used. Appropriate secondary antibodies were used and detection was performed with FastRed TR/Naphthol (Sigma) resulting in red staining of antigenexpressing cells.

\section{RNA isolation and quantitative reverse transcription-PCR}

RNA was isolated from hind paws using Trizol (Invitrogen, Grand Island, NY, USA). Quantitative reverse transcriptasePCR was performed using SYBR Green. The expression of the target molecules was normalised to the expression of $\beta$-actin. Untreated wild-type mice served as reference for comparison between groups and no amplification factor was used for relative mRNA calculation. The data are expressed as arbitrary units. Details of the primers for $\beta$-actin, osteocalcin, Ihh, transforming 
growth factor (TGF)- $\beta$, BMP2, Sox9, Runx2, Smad3, periostin, gli-2, osterix and collagen type $\mathrm{X}$ can be found in the online supplementary table 1 .

\section{ELISA}

Serum was analysed using commercially available ELISA kits for osteocalcin, TGF $\beta$ (both R\&D Systems, Minneapolis, Minnesota, USA) and collagen type II cleavage products (Teco, Sissach, Switzerland).

\section{Micro-CT}

Right tibial bones were fixed overnight in $4.0 \%$ paraformaldehyde and stored in 70\% ethanol before micro-CT analysis with a high-resolution CT scanner (GE explore Locus SP Specimen Scanner; GE Healthcare, London, Canada). Bones were placed in a sealed acrylic specimen holder containing phosphate-buffered saline and a hydroxyapatite phantom, which was used to calibrate Hounsfield units ( $\mathrm{HU})$ to mineral density $(\mathrm{mg} / \mathrm{ml})$. After scanning, images were reconstructed to an isotropic voxel size
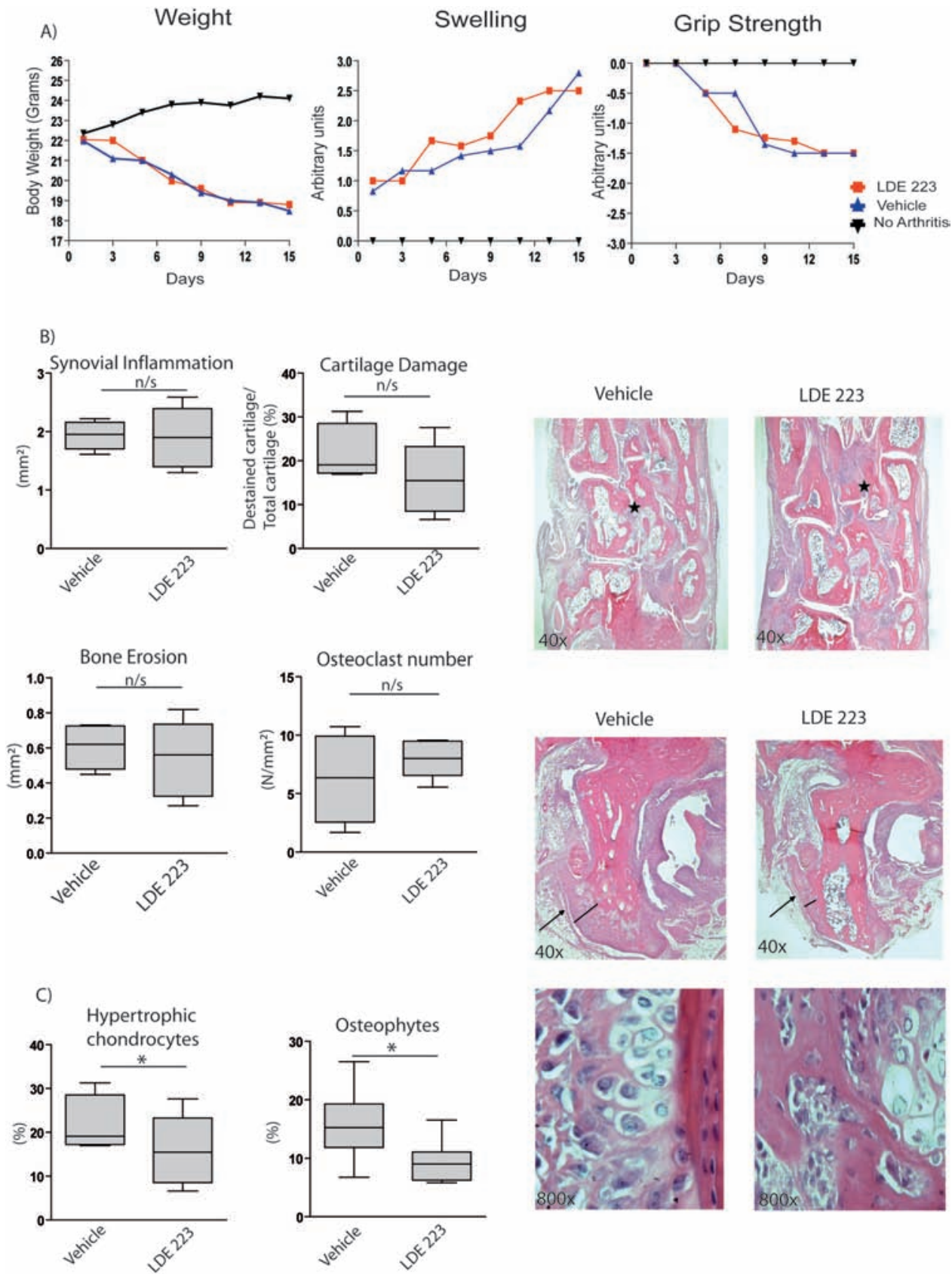

Figure 1 Blockade of Smo does not affect joint inflammation but specifically blocks osteophyte formation. (A) Body weight, joint swelling and grip strength of control mice ( $N=6$; black curves) and mice after serum transfer (serum-induced arthritis $(S I A)$; $N=6)$ and treatment with the Smo inhibitor LDE223 $(N=6$; red) or vehicle $(N=6$; blue). (B) Histomorphometric analysis of hind paws for synovial inflammation, cartilage damage, bone erosion and osteoclast numbers. Microphotographs show haematoxylin and eosin (H\&E) stained hind paw sections. Stars show areas of inflammation and bone erosion (Original magnification $40 \times$ ). (C) Histomorphometric analysis of hypertrophic chondrocytes and osteophytes. Microphotographs indicate H\&E stained hind paw sections showing osteophytes along the calcaneal bone (original magnification $40 \times$ upper panel; $800 \times$ : lower panel). Arrows show hypertrophic chondrocyte areas and bars indicate osteophytes width. Data are the medians (bars), 25th and 75th centile (box) and range (whiskers: minimums and maximum). Asterisks indicate significance $(p<0.05)$. 
of $13.2 \mu \mathrm{m}^{3}$ using a standard Feldkamp Conebeam algorithm and a threshold of $585 \mathrm{mg} / \mathrm{ml}$.

\section{Statistical analysis}

Data are presented as the mean \pm SEM, when Gaussian distribution was present or as median (range) when Gaussian distribution was absent. For group comparison, we used oneway factorial analysis of variance with the ANOVA test or the Mann-Whitney test (when not normally distributed). A p value $<0.05$ was considered significant.

\section{RESULTS}

\section{Blockade of Smo does not affect joint inflammation but specifically blocks osteophyte formation}

We first tested whether blockade of Hh pathway by targeting Smo inhibits arthritis. Clinical symptoms of disease developed quickly 2-3 days after serum transfer and resulted in significant weight loss, joint swelling and reduction in grip strength. No signs of arthritis were seen in the control group. Blockade of Smo had no effect on any of the aforementioned clinical signs of the disease (figure 1A).

To investigate the effects of Hh inhibition in more detail, we performed histological analyses of the hind paws 14 days after arthritis induction. Mice exposed to serum transfer showed prominent synovial infiltration, bone erosion and cartilage damage. We could not detect any effect of Smo inhibition on the quantity of the synovial inflammatory infiltrates (median area of inflammation: vehicle: $1.95 \mathrm{~mm}^{2}$ vs LDE223: $1.9 \mathrm{~mm}^{2}, \mathrm{p}=0.84$ ). Also, there was no difference in the cellular composition of macrophages, neutrophils, $T$ lymphocytes and B lymphocytes (data not shown). Moreover, both vehicle- and LDE223- treated mice showed a similar amount of proteoglycan loss of the articular cartilage (median percentage destained cartilage: vehicle: 19.08\% vs LDE223: $15.49 \%$, p=0.28), osteoclast differentiation and bone erosion (median erosion area: vehicle: $0.62 \mathrm{~mm}^{2}$ vs LDE223: $0.56 \mathrm{~mm}^{2}, \mathrm{p}=0.60$ ) (figure 1B).

As with many other models of arthritis, osteophyte formation is a key feature of serum transfer arthritis. Lesions usually emerge at sites exposed to mechanical stress such as the calcaneal bone and the tendon insertion sites of tarsal bones. We quantitatively assessed osteophytes and found large lesions in vehicle-treated mice (median percentage of tarsal bone covered by osteophytes: $15.27 \%$ ). No osteophytes were found in the negative control group which was not induced for arthritis, clearly indicating that these lesions are specifically linked to arthritis. Smo inhibition significantly inhibited the development of osteophytes (median percentage of tarsal bone covered by osteophytes: $9.02 \%, p=0.001)$. As hypertrophic chondrocytes are considered important for osteophyte formation, we also analysed the relative numbers of hypertrophic chondrocytes within osteophytes in untreated and LDE223-treated animals. Smo blockade yielded significantly reduced numbers of hypertrophic chondrocytes in osteophytes (median percentage of hypertrophic chondrocytes: vehicle: $15.22 \%$ vs LDE223: 8.60\%, p=0.001) (figure 1C).

\section{Irregular periosteal bone apposition was normalised after blockade of Smo}

We next specifically assessed bone formation at periosteal and trabecular skeletal sites of the tibial bone by measuring mineral apposition using calcein labelling. Whereas, there was homogeneous bone apposition at cortical sites of non-arthritic controls (figure 2A), serum transfer-induced arthritis led to enhanced as
A)

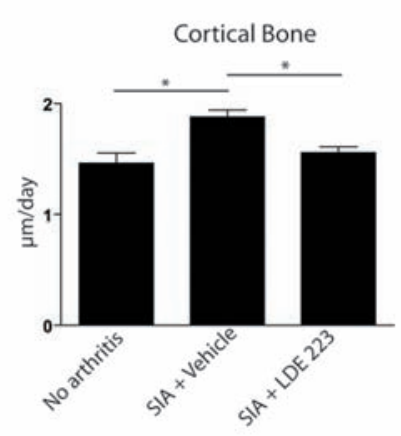

B)

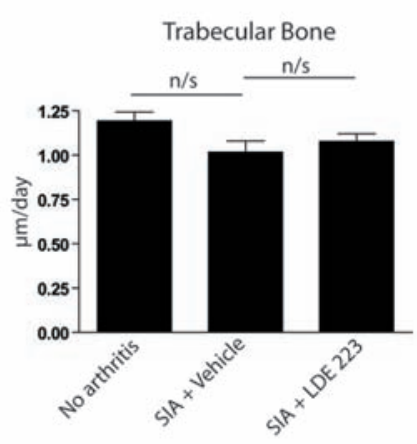

No arthritis

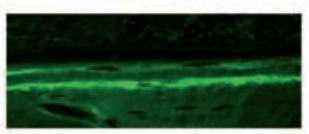

SIA + Vehicle

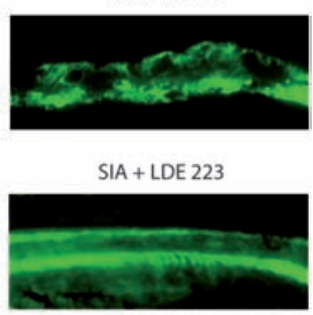

No arthritis

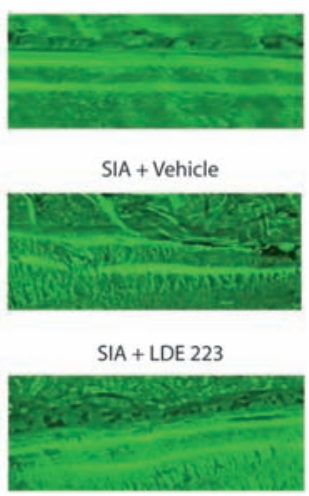

Figure 2 Increased and irregular periosteal mineral apposition in arthritis is reversed by blockade of Smo. (A) Calcein labelling by fluorescence microscopy showing mineral apposition rate ( $\mu \mathrm{m} /$ day) in the cortical area. (B) Calcein labelling by fluorescence microscopy showing mineral apposition rate $(\mu \mathrm{m} /$ day $)$ in the trabecular area. Data are the mean \pm SEM. Asterisks indicate statistical significance $(p<0.05)$. SIA, serum-induced arthritis.

well as irregular patchy apposition of bone, particularly close to the ankle joint, indicating that arthritis affects the anatomy and function of the periosteal bone area. This effect was normalised by Smo blockade (figure 2A). In contrast, arthritis did not enhance bone formation in the trabecular bone compartment but rather led to a small decrease of bone formation, which did not reach statistical significance (figure 2B). A very similar pattern was also seen in mice treated with LDE223, suggesting that Smo blockade specifically inhibits cortical bone changes in arthritis. The lack of an effect of Smo blockade on trabecular bone is also highlighted by a detailed analysis of the trabecular bone architecture showing no differences between vehicle and LDE223 treated mice (online supplementary figure 1).

\section{Blockade of Smo rescues arthritis-induced upregulation of chondrocyte and osteoblast differentiation genes}

We next characterised the molecular effects of Smo blockade in vivo by quantitatively analysing the mRNA expression of genes involved in chondrocyte and osteoblast differentiation in arthritic joints. As compared with non-arthritic controls, we found significant upregulation of genes involved in chondrocyte differentiation and early endochondral ossification such as Ihh, Smad3, Sox9, TGF $\beta$ and BMP2 in the joints of vehicletreated arthritic mice (figure 3A-E). Importantly, Smo blockade completely abrogated the upregulation of Ihh, Smad3, Sox9, TGF $\beta$ and BMP2 and mRNA levels were indistinguishable from 
A)

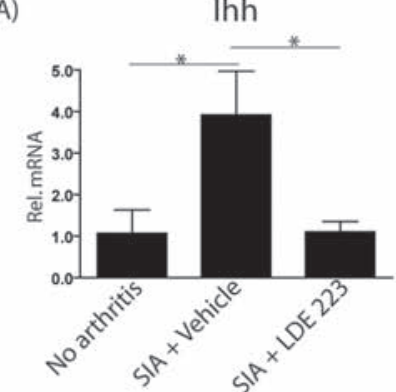

C)

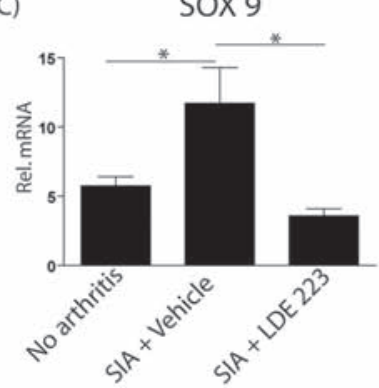

E)

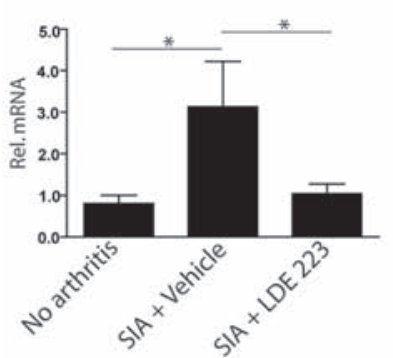

G)

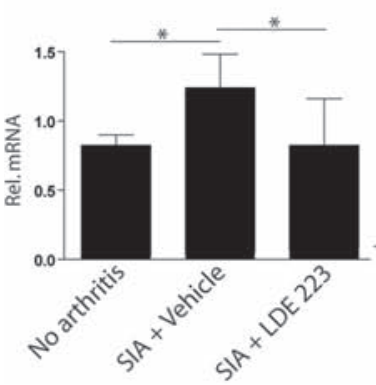

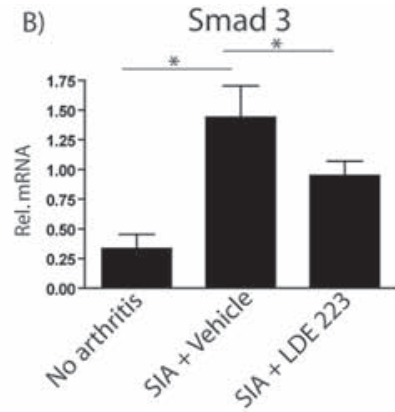

D)

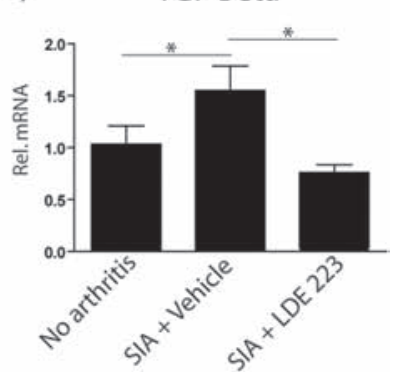

ค)

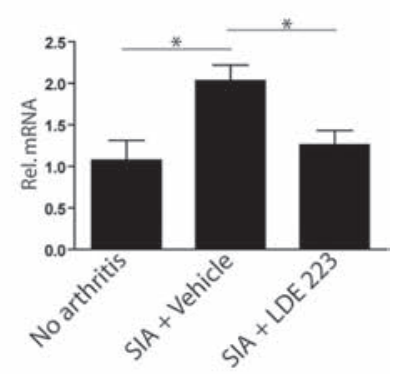

H)

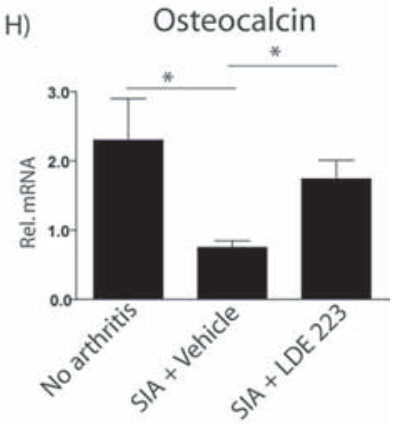

Figure 3 Blockade of Smo rescues arthritis-induced upregulation of chondrocyte and osteoblast differentiation genes. RT-PCR analysis of joint tissue from non-arthritic control mice and mice induced for serumtransfer arthritis treated with either vehicle or LDE223 for 2 weeks. (A) Ihh, (B) Smad3, (C) Sox9 (D) TGF $\beta$, (E) BMP2, (F) Runx2, (G) osterix and $(\mathrm{H})$ osteocalcin. Data are the mean \pm SEM. Asterisks indicate statistical significance $(p<0.05)$. SIA, serum-induced arthritis.

non-arthritic control mice (figure 3A-E). When analysing the expression of markers of osteoblast differentiation, we found that Runx2 and osterix were significantly increased in arthritic joints as compared with non-arthritic controls (figure 3F-G). Again, this effect was efficiently inhibited by blocking Smo. Interestingly, osteocalcin, a marker for osteoblast function, was significantly downregulated during autoantibody-induced arthritis: osteocalcin expression was almost three times reduced in arthritic animals as compared with non-arthritic controls.

This downregulation was rescued in arthritic mice subject to blockade of Smo.

\section{Periosteal activation and chondrocyte hypertrophy in arthritis} depend on hedgehog signalling

Based on the inhibitory effects of Hh blockade on osteophyte formation we considered that reactivation of this developmental signalling pathway is pivotal for endochondral ossification and periosteal activation. Expression of collagen X, a specific marker for hypertrophic chondrocytes was virtually absent in nonarthritic mice but strongly increased in vehicle-treated arthritic mice (figure 4A). After Smo blockade, however, no expression of collagen X mRNA and protein could be detected, suggesting complete abrogation of chondrocyte hypertrophy after Hh inhibition (figure 4D). Moreover, also, the downstream transcription factor of Smo, Gli2, a marker of hedgehog signalling activation, ${ }^{20}$ was expressed both on protein and mRNA level at sites of hypertrophic cartilage formation. Gli2 expression was also blocked when inhibiting Smo, by LDE223. Furthermore, collagen X and Gli2 expression at sites of osteophyte formation was accompanied by periosteal activation as mirrored by induced expression of periostin (figures 4B,C,E,F). Periostin is expressed by developing osteoblasts and its expression is induced by both TGF $\beta$ and BMP2. ${ }^{21}$ The virtually complete inhibition of $\mathrm{mRNA}$ expression of periostin and the partial inhibition of periostin protein expression after blockade of Smo suggest an intensive interaction between Hh-induced hypertrophic cartilage formation and osteoblastogenesis.

\section{Smo directly regulates cartilage and bone metabolism}

We also aimed to assess whether the effect of Smo inhibition can be seen by assessing systemic markers of cartilage and bone metabolism. We measured TGF $\beta$ serum levels by ELISA, as it is an important contributor to chondrocyte hypertrophy and bone formation. Interestingly, TGF $\beta$ levels were increased in vehicle-treated arthritic mice (mean \pm SD serum level $72.63 \pm$ $9.52 \mathrm{pg} / \mathrm{ml}$ ) as compared with non-arthritic controls (55.39 \pm $8.98 \mathrm{pg} / \mathrm{ml} \mathrm{p}=0.031)$. Moreover, Smo blockade reversed elevated TGF $\beta$ levels $(57.47 \pm 7.81 \mathrm{pg} / \mathrm{ml} \mathrm{p}=0.017)$ (figure $5 \mathrm{~A}$ ). We also measured collagen type II cleavage products by ELISA. Interestingly, we found elevated levels of collagen II turnover in vehicle-treated arthritic mice (mean \pm SD serum level $416.5 \pm 36$ $\mathrm{pg} / \mathrm{ml}$ ) as compared with non-arthritic controls $279.2 \pm 15 \mathrm{pg} /$ $\mathrm{ml}, \mathrm{p}=0.041$ ), indicating a state of active cartilage metabolism (figure 5B). Again, inhibition of Smo reversed elevated cartilage metabolism $(299 \pm 14 \mathrm{pg} / \mathrm{ml} \mathrm{p}=0.049)$. At the same time, serum levels of osteocalcin, which were decreased in vehicle-treated arthritic mice compared with healthy controls (mean \pm SD serum levels $44.22 \pm 7.98 \mathrm{ng} / \mathrm{ml}$ vs $19.65 \pm 7.87, \mathrm{p}=0.0043$ ), were significantly increased $(34.92 \pm 12.26 \mathrm{ng} / \mathrm{ml} \mathrm{p}=0.0043$ compared with vehicle-treated arthritic mice) after 2 weeks of treatment (figure 5C).

\section{DISCUSSION}

In this study we show a central role of the Hh pathway in osteophyte formation during inflammatory arthritis. By using a specific inhibitor against the key Hh signalling component Smo we (1) significantly reduced osteophyte formation within 2 weeks of onset of arthritis but (2) did not affect the inflammatory signs of arthritis and (3) did not induce a negative effect on systemic bone turnover and trabecular bone architecture. Molecular analyses showed that Smo blockade affected endochondral ossificationthat is, the formation of hypertrophic chondrocytes, which are directly involved in the process of osteophyte formation. 

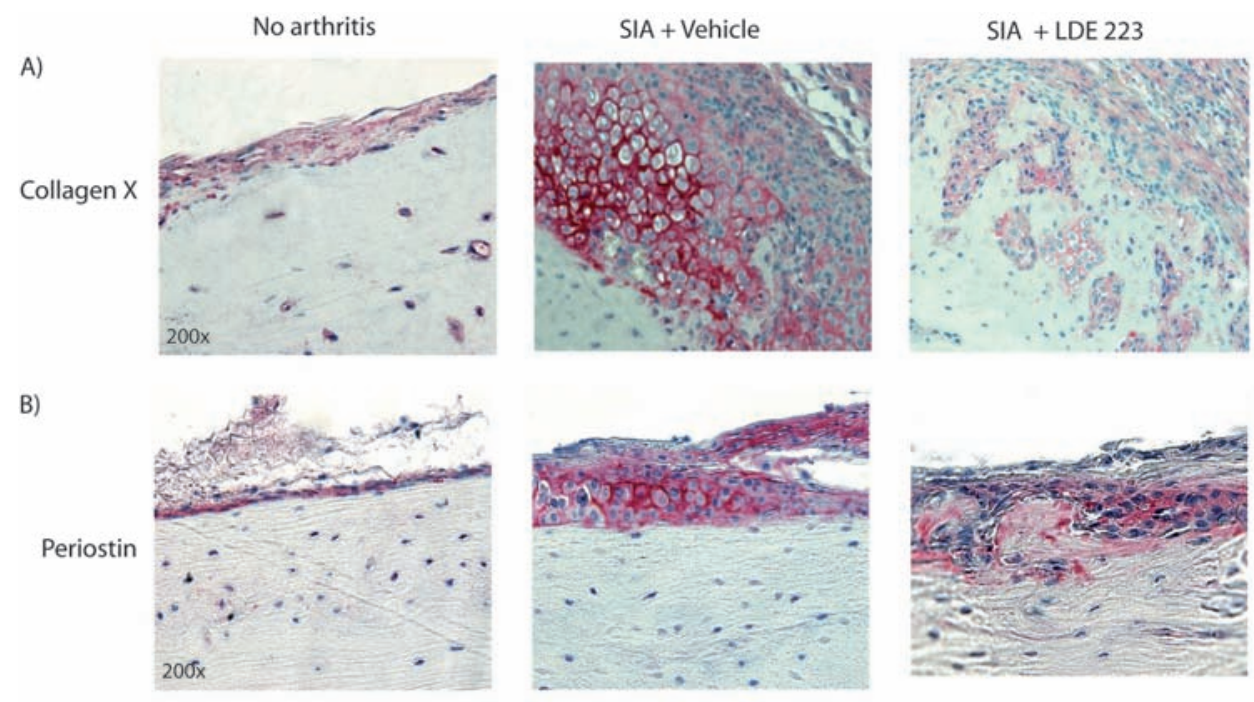

C)
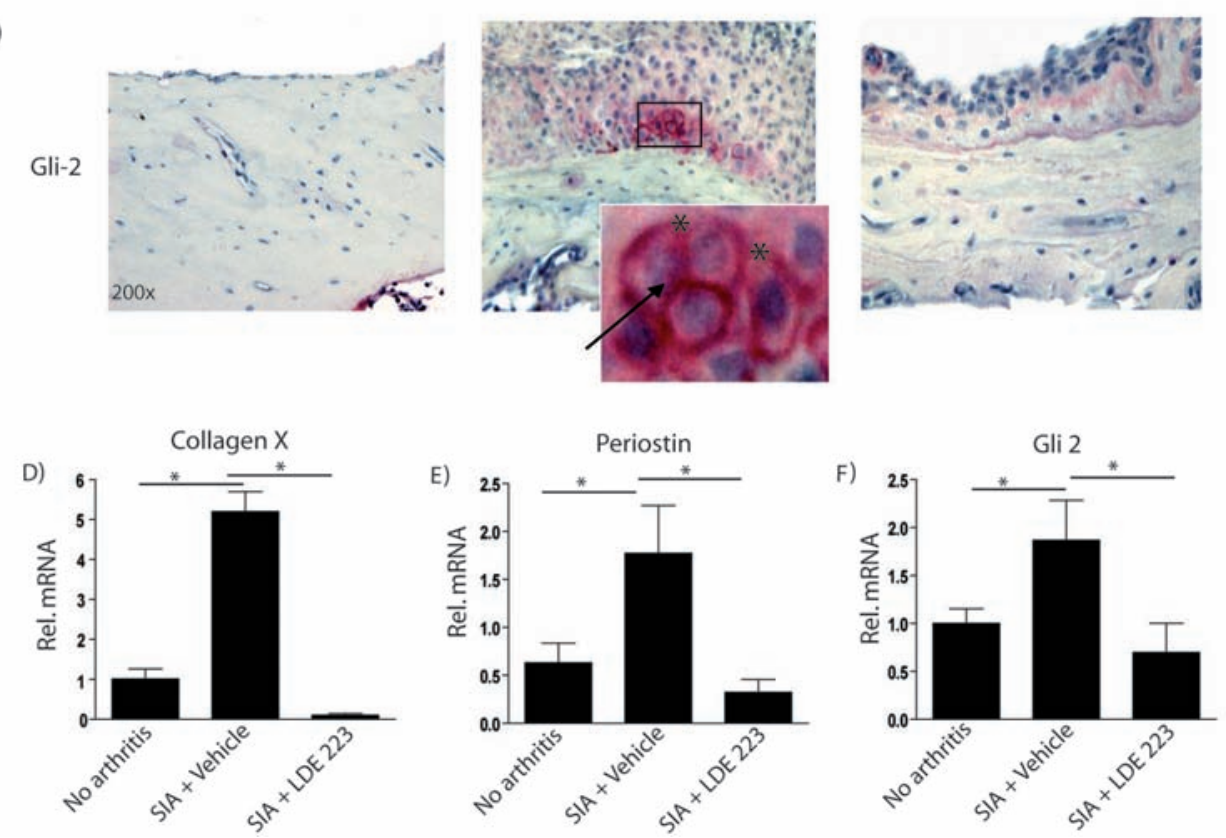

Figure 4 Periosteal activation and chondrocyte hypertrophy in arthritis depend on hedgehog signalling. (A-C) Immunohistochemistry of the periosteal area of paws from non-arthritic control mice and mice induced for serum-transfer arthritis treated with either vehicle or LDE223 for collagen $X$, periostin and Gli2 on untreated animals and normalisation after Smo pharmacological blockade during 2 weeks. Higher magnification $(\times 1000)$ of Gli2 staining show nuclear and cytoplasmic expression. (Arrow indicates nuclear staining and stars indicate cytoplasmic staining.) (D-F) RT-PCR analysis of joint tissue for relative mRNA levels of collagen $\mathrm{X}$, periostin and Gli2. Data are the mean $\pm \mathrm{SEM}$. Asterisks indicate statistical significance $(p<0.05)$. SIA, serum-induced arthritis.

Joint diseases with the exception of rheumatoid arthritis are typically characterised by bony spur formation, which emerges from the periarticular periosteal surface. This process can be seen as an intrinsic reaction pattern of joints towards mechanical and/ or inflammatory stress. Bony spurs thus represent an overshooting reaction of the joint building bony bridges, which stabilise the affected region. At the same time bony spur formation is part of a pathological process, which leads to ankylosis and loss of function of the affected joint. Osteophytes are based on new bone formation, which occurs locally at the site of inflammation, particularly when inflammation coincides with regions exposed to high mechanical load such as the entheses. Importantly, new bone formation in conjunction with osteophytes is a local process which contrasts systemic loss of trabecular bone in inflammatory disease. Thus, about $40-60 \%$ of patients affected by ankylosing spondylitis show osteopenia, an indicator of trabecular bones loss. Moreover, osteoporosis and fracture risk are increased in ankylosing spondylitis despite abundance of new bone formation along the periosteal sites..$^{22-24}$ These clinical observations demonstrate that highly divergent processes are occurring in the outer cortical bone surface, which shows a net increase of bone, and the inner trabecular skeletal compartment, which is subject to net bone loss. As a consequence, factors have to be identified which specifically trigger new bone formation at periosteal sites, as only inhibition of such factors may provide a therapeutic rationale to interfere with osteophyte formation without precipitating further trabecular bone loss.

Generation of osteophytes requires periosteal activation, which is highlighted by the proliferation and differentiation of mesenchymal cells building the periosteum. These cells 
A)

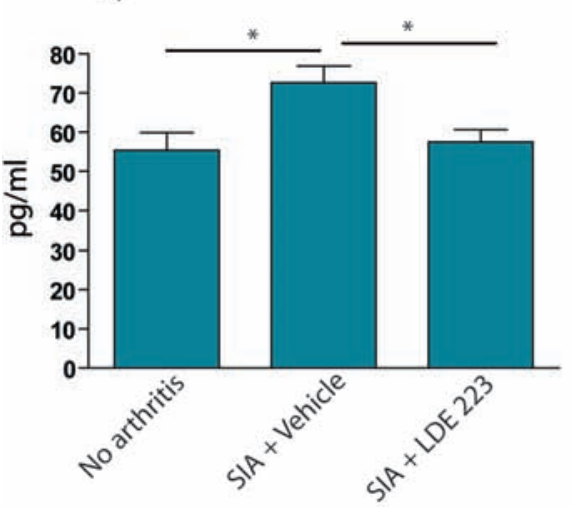

B)

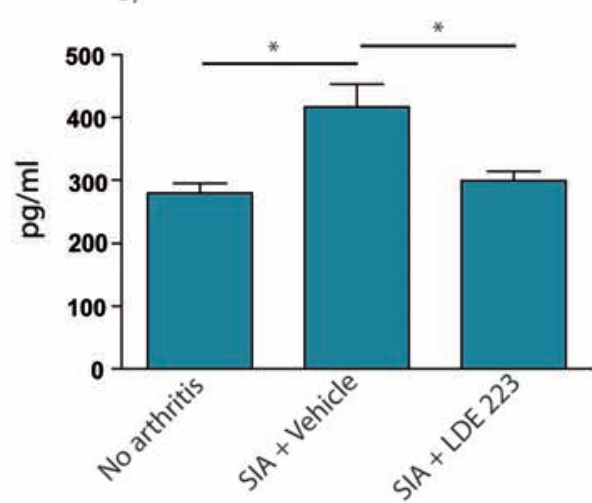

Osteocalcin

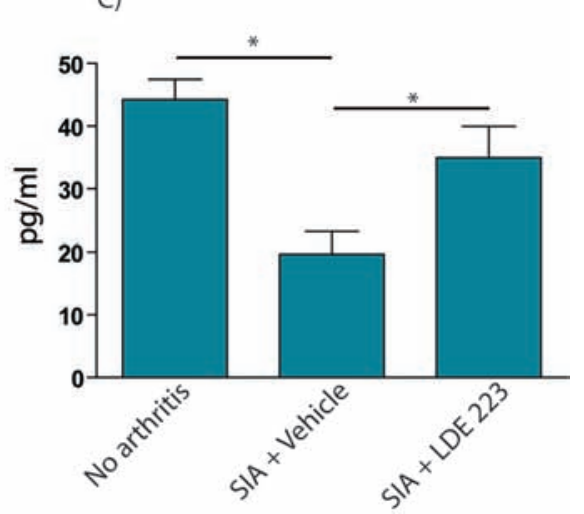

Figure 5 Smoothened directly regulates cartilage and bone metabolism. Serum analysis by ELISA for (A) transforming growth factor $\beta$ (TGF $\beta$ ); (B) collagen type 2 cleavage products and (C) osteocalcin in non-arthritic control mice and mice induced for serum-transfer arthritis treated with either vehicle or LDE223 Data are the mean \pm SEM. Asterisks indicate statistical significance $(p<0.05)$. SIA, serum-induced arthritis.

differentiate into hypertrophic chondrocytes and also into osteoblasts depositing mineralised extracellular matrix. This process of endochondral ossification is also found in the growth plates and is an essential process in the development and growth of long bones. Classically inflammatory mediators such as tumour necrosis factor $\alpha$ (TNF $\alpha$ ) and interleukin 1 are not the main triggers for new bone formation but rather induce bone loss. Thus inflammatory cytokines are not responsible for osteophyte formation but can be regarded as precipitators for enhanced bone loss. ${ }^{25}$ For instance, TNF induces osteoclast differentiation and enhances bone resorption. At the same time TNFo inhibits bone formation by stimulating the expression of proteins that interfere with the Wnt signalling pathway, which is an essential mediator of osteoblast differentiation. In accordance, blockade of an inflammatory cytokine such as TNF $\alpha$ but also factors involved in osteoclastogenesis do not halt new bone formation in animal models of arthritis or in human disease. ${ }^{26-29}$

Recently, growing scientific interest has been directed towards the role of molecules involved in bone formation in the pathogenesis of osteophytes. Different anabolic pathways such as TGF $\beta$, BMPs and Wnt proteins have been described as inducing new bone formation and building up osteophytes during the course of arthritis. ${ }^{6930}$ However, as these pathways are also fundamentally involved in physiological bone remodelling, inhibition of these pathways may precipitate osteoporosis and may thus not be a feasible therapeutic strategy in inflammatory diseases, which already show an increased risk of osteoporosis. Some of the molecular processes involved in osteophyte formation, however, may be specific and may not play a role in physiological bone remodelling of the adult skeleton but rather may reactivate old developmental programmes regulating bone growth.

The Hh signalling pathway is one of the fundamental signal transduction pathways in embryonic development. Hh proteins bind to the receptor Ptch, which releases Smo, promoting the activation of the transcription factors Gli1, Gli2 and Gli3. Among them, Gli2 and Gli3 are the primary responders, whereas Gli1 has been described acting to amplify the response. Moreover, Gli2 appears to function predominantly as a transcriptional activator in vivo, ${ }^{19}$ whereas Gli3 functions primarily as a repressor. In mammals, three Hh proteins are known: sonic hedgehog (Shh), which is involved in the neural tube formation and in the morphogenesis of various organs including the axial skeleton, limbs, lungs, skin, hair and teeth, and is also a regulator of the development of hair follicles and sebaceous glands. Desert hedgehog (Dhh) has been described as regulating spermatogenesis and helping in the organisation of the perineurium. Finally, Indian hedgehog (Ihh) which coordinates proliferation and maturation of chondrocytes during the development of the endochondral skeleton. Ihh is expressed in prehypertrophic chondrocytes and regulates the development to hypertrophic differentiation by negative regulation of parathyroid hormone related protein (PTHrP). PTHrP acts through its receptor Pthr1 inhibiting chondrocyte hypertrophy and suppressing Ihh expression keeping chondrocytes in a proliferating state. ${ }^{31} 32$ Recent studies have shown a role for Hh in hypertrophic chondrocyte formation in the cartilage of experimental osteoarthritis $(\mathrm{OA}) \cdot{ }^{14} \mathrm{Hh}$ is activated in human and mouse OA, and cartilage in OA shows high expression of Hh targets, such as Gli1, Ptch1 and Hhip associated with chondrocyte hypertrophy and collagen $\mathrm{X}$ expression. Interestingly, activation of Hh signalling in chondrocytes caused a more severe osteoarthritic phenotype in a surgical model of OA. These data demonstrated the role of Hh in adult tissue-that is, in pathological changes of the cartilage associated with chondrocyte hypertrophy.

In our study, we demonstrate a specific effect of Smo inhibition on osteophyte formation, suggesting a central involvement of the Hh pathway in the process of bony spur formation during arthritis. Milder, but not significant, cartilage-protective effects of $\mathrm{Hh}$ targeting were also found, which reinforce the aforementioned concept that chondrocyte hypertrophy is a process involved in cartilage destruction. The lack of effect on inflammation is important as it shows that inhibition of Smo directly acts on osteophytes and that the observed effects are not indirect owing to blunted inflammation. Moreover, there was no enhancing effect on osteoclast formation and bone erosion upon Hh blockade. Cross-talk between Wnt proteins and BMPs and the RANKL/OPG system has been described: activation of the Wnt pathway blocks bone resorption through upregulation of OPG. ${ }^{33}$ Thus, interference with the Wnt pathway, but also with BMP signalling, which share common intracellular targets, might enhance bone resorption. In contrast, Smo inhibition did not yield any effect on bone erosion. This finding could be based on the fact that Hh signalling itself can enhance osteoclastogenesis and bone resorption via the upregulation of PTHrP and Crebmediated RANKL transcription, ${ }^{33} 34$ which is abolished when the Hh pathway is disrupted. 
Moreover, no negative effects on systemic bone architecture and bone formation in the trabecular bone compartment were seen. Hh targeting even reversed the decrease of osteocalcin expression as well as the drop in osteocalcin serum levels seen in arthritis, indicating that no systemic suppression of bone formation occurs when Hh signalling is blocked. The virtually complete inhibition of periostin expression after blockade of Smo, however, suggests an intensive interaction between $\mathrm{Hh}$-induced hypertrophic chondrocyte formation and local periosteal osteoblastogenesis, which is required for the remodelling of chondrocytic regions of the osteophyte into new bone.

In summary, we conclude that targeting of the Hh signalling pathway by inhibition of the central signalling component Smo specifically blocks osteophyte formation in inflammatory arthritis without precipitating local or systemic bone loss. The high potential of Hh targeting in blocking chondrocyte hypertrophy and the central role of endochondral ossification in the emergence of osteophytes are the reasons why Hh inhibition influences osteophyte formation in arthritis. This concept provides a unique chance of developing therapeutic strategies to specifically target osteophyte formation and to modify the periosteal response and prevent ankylosis in arthritis.

Acknowledgements The authors thank Professor Klaus von der Mark from the University of Erlangen-Nuremberg for providing the anti collagen $\mathrm{X}$ antibody, and Isabel Schmidt, Eugenia Schefler, Cornelia Stoll and Martin Steffen for excellent technical assistance.

Funding This study was supported by the Deutsche Forschungsgemeinschaft (FG 661/ TP4 and SPP1468-IMMUNOBONE; to AH, GS and JZ), the Bundesministerium für Bildung und Forschung (ANCYLOSS and IMMUNOPAIN; to GS, JZ and AH), the MASTERSWITCH, KINACEPT and ADIPOA projects of the European Union (to GS), the Interdisciplinary Centre for Clinical Research and the ELAN fund of the University of Erlangen-Nuremberg.

Competing interest None.

Provenance and peer review Not commissioned; externally peer reviewed.

\section{REFERENCES}

1. Benjamin M, McGonagle D. The anatomical basis for disease localisation in seronegative spondyloarthropathy at entheses and related sites.

J Anat 2001;199(Pt 5):503-26.

2. Maksymowych WP. Progress in spondylarthritis. Spondyloarthritis: lessons from imaging. Arthritis Res Ther 2009;11:222.

3. Lories RJ, Luyten FP, de Vlam K. Progress in spondylarthritis. Mechanisms of new bone formation in spondyloarthritis. Arthritis Res Ther 2009;11:221.

4. van der Kraan PM, van den Berg WB. Osteophytes: relevance and biology. Osteoarthr Cartil 2007;15:237-44.

5. Bianco P, Cancedda FD, Riminucci M, et al. Bone formation via cartilage models: the "borderline" chondrocyte. Matrix Biol 1998;17:185-92.

6. Scharstuhl A, Glansbeek HL, van Beuningen HM, et al. Inhibition of endogenous TGF-beta during experimental osteoarthritis prevents osteophyte formation and impairs cartilage repair. J Immunol 2002;169:507-14.

7. Lories RJ, Derese I, Luyten FP. Modulation of bone morphogenetic protein signaling inhibits the onset and progression of ankylosing enthesitis. $J$ Clin Invest 2005;115:1571-9.

8. Diarra D, Stolina M, Polzer K, et al. Dickkopf-1 is a master regulator of joint remodeling. Nat Med 2007;13:156-63.

9. Nakase T, Miyaji T, Tomita T, et al. Localization of bone morphogenetic protein-2 in human osteoarthritic cartilage and osteophyte. Osteoarthr Cartil 2003;11:278-84
10. Day TF, Yang Y. Wnt and hedgehog signaling pathways in bone development. J Bone Joint Surg Am 2008;90 Suppl 1:19-24.

11. Chung UI, Schipani E, McMahon AP, et al. Indian hedgehog couples chondrogenesis to osteogenesis in endochondral bone development. J Clin Invest 2001;107:295-304.

12. Wilson CW, Chuang PT. Mechanism and evolution of cytosolic Hedgehog signal transduction. Development 2010;137:2079-94.

13. Pasca di Magliano M, Hebrok M. Hedgehog signalling in cancer formation and maintenance. Nat Rev Cancer 2003;3:903-11.

14. Lin AC, Seeto BL, Bartoszko JM, et al. Modulating hedgehog signaling can attenuate the severity of osteoarthritis. Nat Med 2009;15:1421-5.

15. Maccioni M, Zeder-Lutz G, Huang $\mathrm{H}$, et al. Arthritogenic monoclonal antibodies from K/BxN mice. J Exp Med 2002;195:1071-7.

16. Peukert S, Jain RK, Geisser A, et al. Identification and structure-activity relationships of ortho-biphenyl carboxamides as potent smoothened antagonists inhibiting the hedgehog signaling pathway. Bioorg Med Chem Lett 2009;19:328-31.

17. Polzer K, Schett G, Zwerina J. The lonely death: chondrocyte apoptosis in TNFinduced arthritis. Autoimmunity 2007; 40:333-6.

18. Zwerina J, Hayer S, Tohidast-Akrad M, et al. Single and combined inhibition of tumor necrosis factor, interleukin-1, and RANKL pathways in tumor necrosis factor-induced arthritis: effects on synovial inflammation, bone erosion, and cartilage destruction. Arthritis Rheum 2004;50:277-90.

19. Parfitt AM, Drezner MK, Glorieux FH, et al. Bone histomorphometry: standardization of nomenclature, symbols, and units. Report of the ASBMR Histomorphometry Nomenclature Committee. J Bone Miner Res 1987;2:595-610.

20. Joeng KS, Long F. The Gli2 transcriptional activator is a crucial effector for Ihh signaling in osteoblast development and cartilage vascularization. Development 2009;136:4177-85.

21. Horiuchi K, Amizuka N, Takeshita S, et al. Identification and characterization of a novel protein, periostin, with restricted expression to periosteum and periodontal ligament and increased expression by transforming growth factor beta. J Bone Miner Res 1999;14:1239-49.

22. Maillefert JF, Aho LS, El Maghraoui A, et al. Changes in bone density in patients with ankylosing spondylitis: a two-year follow-up study. Osteoporos Int 2001;12:605-9.

23. Roux C. Osteoporosis in inflammatory joint diseases. Osteoporos Int 2011;22:421-33

24. Karberg K, Zochling J, Sieper J, et al. Bone loss is detected more frequently in patients with ankylosing spondylitis with syndesmophytes. J Rheumatol 2005:32:1290-8.

25. Schett G, Teitelbaum SL. Osteoclasts and Arthritis. J Bone Miner Res 2009;24:1142-6

26. Schett G, Stolina M, Dwyer D, et al. Tumor necrosis factor alpha and RANKL blockade cannot halt bony spur formation in experimental inflammatory arthritis. Arthritis Rheum 2009;60:2644-54.

27. Lories RJ, Matthys P, de Vlam K, et al. Ankylosing enthesitis, dactylitis, and onychoperiostitis in male DBA/1 mice: a model of psoriatic arthritis. Ann Rheum Dis 2004;63:595-8.

28. Lories RJ, Derese I, Luyten FP. Inhibition of osteoclasts does not prevent joint ankylosis in a mouse model of spondyloarthritis. Rheumatology (Oxford) 2008:47:605-8.

29. van der Heijde D, Landewé R, Einstein S, et al. Radiographic progression of ankylosing spondylitis after up to two years of treatment with etanercept. Arthritis Rheum 2008;58:1324-31.

30. Heiland GR, Zwerina K, Baum W, et al. Neutralisation of Dkk-1 protects from systemic bone loss during inflammation and reduces sclerostin expression. Ann Rheum Dis 2010;69:2152-9.

31. Mak KK, Kronenberg HM, Chuang PT, et al. Indian hedgehog signals independently of PTHrP to promote chondrocyte hypertrophy. Development 2008;135:1947-56.

32. Mak KK, Chen MH, Day TF, et al. Wnt/beta-catenin signaling interacts differentially with Ihh signaling in controlling endochondral bone and synovial joint formation. Development 2006;133:3695-707.

33. Glass DA 2nd, Bialek P, Ahn JD, et al. Canonical Wnt signaling in differentiated osteoblasts controls osteoclast differentiation. Dev Cell 2005;8:751-64.

34. Mak KK, Bi Y, Wan C, et al. Hedgehog signaling in mature osteoblasts regulates bone formation and resorption by controlling PTHrP and RANKL expression. Dev Cell 2008:14:674-88. 


\section{ARD Blockade of the hedgehog pathway inhibits osteophyte formation in arthritis}

Gisela Ruiz-Heiland, Angelika Horn, Pawel Zerr, Willy Hofstetter, Wolfgang Baum, Michael Stock, Jörg H Distler, Falk Nimmerjahn, Georg Schett and Jochen Zwerina

Ann Rheum Dis 2012 71: 400-407 originally published online January 10, 2012

doi: 10.1136/ard.2010.148262

Updated information and services can be found at:

http://ard.bmj.com/content/71/3/400

These include:

Supplementary Material

References

Email alerting service
Supplementary material can be found at:

http://ard.bmj.com/content/suppl/2011/06/22/ard.2010.148262.DC1.ht $\mathrm{ml}$

This article cites 34 articles, 10 of which you can access for free at: http://ard.bmj.com/content/71/3/400\#BIBL

Receive free email alerts when new articles cite this article. Sign up in the box at the top right corner of the online article.

Collections

Articles on similar topics can be found in the following collections

Degenerative joint disease (4621)

Musculoskeletal syndromes (4930)

Immunology (including allergy) (5116)

Inflammation (1244)

\section{Notes}

To request permissions go to:

http://group.bmj.com/group/rights-licensing/permissions

To order reprints go to:

http://journals.bmj.com/cgi/reprintform

To subscribe to BMJ go to:

http://group.bmj.com/subscribe/ 